\section{Transfer of persistence effects from frustration produced by blocking to frustration produced by nonreinforcement ${ }^{1}$}

HOWARD GLAZER and ABRAM AMSEL, The University of Texas at Austin, Austin, Tex. 78712

Six groups of rats acquired a response in a runway under conditions of continuous reinforcement, partial reinforcement, or partial goal blocking defined by the presence of an unconsumable food pellet. Extinction was carried out under conditions of either continuous nonreward or continuous blocking. Blocking was as effective as nonreward in producing persistence in extinction. Groups switched from one type of nonreward in acquisition to another type in extinction showed significantly greater persistence compared to nonswitched groups. The results are discussed within a framework of frustration theory in conjunction with the concept of arousal.

A question that has come to the foreground recently in frustration theory is whether or not a single unitary mechanism is involved in the learning of persistent behavior. In this context, three possibilities have been suggested (Amsel, 1969). The most liberal view is that the counterconditioning of any disruptive stimulus $\left(S_{x}\right)$ to an ongoing response $\left(R_{o}\right)$ adds to a nonspecific persistence pool and to the generalized persistence of that organism. A more restricted view holds that there are different, but overlapping, systems controlling persistence, so that there may be some, but not complete, transfer of persistence between such closely related systems as fear and frustration. A third, and most restricted, view holds that specific disrupting stimuli become counterconditioned to specific ongoing responses, and there is no transfer of persistence from one response system to another.

The evidence so far available suggests that the most tenable position is the intermediate one. Studies by Brown \& Wagner (1964), Banks (1967), and Fallon (1968), for example, have shown that there is transfer between persistence derived from frustration and punishment.

Even if persistence is not a single unitary system (that is not to say that it does not depend on a single kind of mechanism), and if transfer of persistence effects is not completely general, it still remains to determine the extent to which transfer from one persistence-producing operation to another does occur. The persistence-producing manipulation whose effects have been studied most is that involving nonreward in partial reinforcement. If, as frustration theory holds, the mechanism mediating persistent behavior following partial reinforcement is $r_{F}-s_{F}-R_{0}$, a question to ask is if persistence is general enough to permit transfer of effects from feedback stimuli of the class, $s_{F}$, produced by different frustration-producing procedures.

One way of approaching the problem is to use a transfer paradigm in which Ss acquire a response under one of two types of frustrating procedure and are then switched to the other frustration-producing procedure in extinction. If different feedback stimuli are assumed to result from the two frustration-producing procedures, it would be expected that, because of stimulus-generalization decrement, Ss switched from one in acquisition to the other in extinction would show less persistence in extinction than would Ss who had both acquired a response and been extinguished under the same frustrating conditions. If, on the other hand, the feedback stimuli are not discriminable, then a switched group should show no less resistance to extinction than would a nonswitched group.

Sixty naive male Wistar albino rats, 70 to 80 days old at the beginning of pretraining, were used in this experiment. APPARATUS

The apparatus consisted of a runway constructed of plywood, painted flat black, and covered with clear Plexiglas. The components of the runway were an 11-in. entry box, an 11 -in. start box, and a 42 -in. runway, all measuring $2-7 / 8$ in. wide and $3-7 / 8$ in. high. Guillotine doors separated the entry and start boxes from the runway proper and also formed a 15 -in. goal box area. The foodcup was a semicircular sheet-metal trough extending the total width of the goal box and was fixed on the end wall of the goal box. The proximal lip of the foodcup was approximately 2 in. above the floor. Slits were made on either side of the last 6 in. of the goal box so that a piece of clear 1/16-in. Plexiglas could be inserted to form a cover over the foodcup. The Plexiglas was highly perforated with 1/16-in. holes. Photoelectric circuitry was used to obtain three $1-\mathrm{ft}$ measures of running time.

\section{PROCEDURE}

The Ss were habituated to a $10 \mathrm{~g}$ food-deprivation schedule for 6 days prior to pretraining and were handled for 2 to 3 min daily during this period. Pretraining consisted of one 3-min period of runway adaptation on each of 3 days, during which groups of two Ss explored simultaneously with the recording circuitry operative. No food was available in the runway or in relation to handling. Ss were trained in the runway under one of three conditions: continuous reinforcement (C), partial $(50 \%)$ reinforcement $(\mathrm{P})$, or partial $(50 \%)$ goal blocking (B). Reinforcement consisted of one 500-mg Noyes pellet. A 500-mg Noyes pellet was also present ${ }^{*}$ in the foodcup on goal-blocked trials. Ss were extinguished under one of two conditions: continuous nonreward $(\mathrm{N})$ or continuous goal blocking (B). On nonreward or blocked trials, Ss remained in the goal box for $20 \mathrm{sec}$. Each $\mathrm{S}$ was given $10 \mathrm{~g}$ of food daily at least $1 / 2 \mathrm{~h}$ after the last trial of the day.

For Ss in Group P and Group PB, the sequence of goal events was determined by a quasirandom schedule in which both rewarded and nonrewarded trials were equally likely to be followed by rewarded or nonrewarded trials. There were four sequences of goal events. Two of these were: $\mathbf{N}, \mathbf{N}, \mathbf{R}, \mathbf{R}, \mathbf{R}, \mathrm{N}, \mathrm{N}, \mathrm{R}, \mathrm{N}, \mathrm{R}, \mathrm{R}, \mathrm{N}, \mathbf{R}, \mathrm{R}, \mathrm{N}, \mathrm{N}$ and $N, R, R, N, R, R, N, N, R, R, N, N, N, R, R, N$. The other two were the same sequences with Rs and Ns reversed. All Ss ran two trials a day, with an intertrial interval of approximately $30 \mathrm{~min}$. Sixty acquisition trials were run followed by 32 extinction trials.

Separate speed (reciprocal-time) measures were obtained for each of the three runway segments. However, analyses of variance on running speed over each segment separately showed essentially the same effects; thus, we are presenting speed data averaged over the three segments of the alley.

\section{Acquisition}

Figure 1 shows speeds for terminal acquisition and extinction. An analysis of variance of the acquisition data showed a significant trials effect $[F(14,756)=591.00, p<.01]$ and $a$ significant Treatment by Trial Blocks interaction $[F(28,756)=2.61, p<.01]$. No other main effects or interactions were found to be significant.

The terminal acquisition data appear as the two points on the left side of Fig. 1. Interpretation of the extinction data becomes complicated if the groups 
Fig. 1. Mean extinction speeds for each group (10 SS) over four trial blocks.

extinguished under different conditions show different terminal acquisition levels. An analysis of variance performed on the last two blocks (eight trials) of acquisition showed no significant main effects or interactions, i.e., no terminal acquisition differences.

\section{Extinction}

The extinction curves in Fig. 1 show that speed decreased significantly over the 32 extinction trials $[F(7,378)=153.35$, $p<.01]$. The main effect of the different extinction procedures was not significant $[F(1,54)=.001]$, nor was there a significant Extinction Condition by Block interaction $[F(7,378)=.87]$. However, the acquisition condition main effect and the Acquisition Condition by Blocks interaction were both found to be significant $[F(2,54)=39.25, p<.01$, and $\mathrm{F}(14,378)=8.57, \mathrm{p}<.01$, respectively], as was the Acquisition Condition by Extinction Condition interaction $[F(2,54)=8.46, p<.01]$. An analysis of variance on the simple effects of acquisition condition was significant for both nonreward and blocking procedures in extinction $[\mathrm{F}(2,54)=22.35, \mathrm{p}<.01$, and $F(2,54)=24.63, p<.01$, respectively]. Pairwise comparisons showed that the switched groups differed significantly from the continuous groups for both extinction procedures (Newman-Keuls, $p<.05$ in all cases). Further comparisons revealed that the difference between the $\mathrm{CN}$ and $\mathrm{PN}$ groups did not differ significantly from the difference between the $\mathrm{CB}$ and $\mathrm{BB}$ groups (Tukey $A, p>.05$ ). Finally, there was also a significant Acquisition Condition by Extinction Condition by Blocks interaction $[F(14,378)=1.94, p<.05]$.

\section{DISCUSSION}

Both the partial-reward and partial-blocking groups were more resistant to extinction than were the continuous groups, and the relative increased persistence of these two partial groups was of about the same magnitude. There is, therefore, no basis to conclude that different amounts of frustration are produced by blocking and nonreward.

An unexpected finding was the significantly greater persistence in extinction of the switched groups (PB and $\mathrm{BN}$ ) over the nonswitched groups (PN and BB). Bacon (1965), employing an L-shaped alley with water reward, found better terminal extinction performance in a group

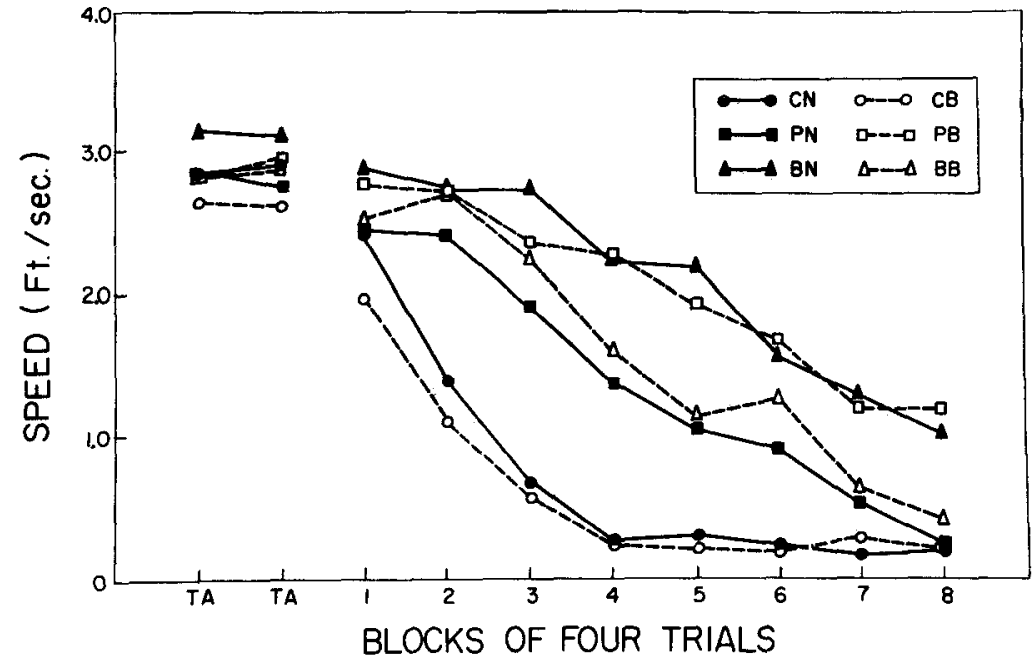

of rats switched from partial blocking in acquisition to nonreward in extinction than in a group trained under partial reward and extinguished under nonreward (nonswitched). While Bacon's blocking procedure consisted of an occlusion of the start box rather than of the goalcup as in the present experiment, the results of both studies suggest generalization of the effects of anticipatory frustration-produced stimuli from one method of producing frustration to another. Still left unexplained, however, is the superior persistence of the switched groups over the nonswitched groups found in the present study and suggested in Bacon's.

If, as the data suggest, the same classically conditioned anticipatory mechanisms operate, and to the same extent, in both the switched and unswitched groups, the explanation of the superior persistence of the switched groups must be sought in terms of a factor affecting the strength of the counterconditioned connection between the frustration-produced stimuli and approach. One way in which the $\mathrm{s}_{\mathrm{F}} \rightarrow$ approach bond could be strengthened might be through an increase in general arousal (Berlyne, 1960) because of the novelty of the frustrating goal event for the switched group. If such arousal has the status of an unconditioned goal response, a conditioned form of the arousal response might serve to strengthen already existing associations, including $\mathrm{SF}_{\mathrm{F}} \rightarrow$ approach. With spaced trials, habituation to novel stimuli proceeds quite slowly (Thompson \& Spencer, 1966), which might account for such an effect of novelty on persistence throughout our extinction phase.

The suggestion that change in stimulation produces greater persistence in extinction is supported by a study by Brown \& Bass (1958), who found that rats extinguished under conditions of variable external stimuli (three different runways) showed significantly greater persistence than did Ss extinguished under conditions of constant stimulation, regardless of whether constant or varied stimulation was encountered in acquisition.

\section{REFERENCES}

AMSEL, A. Behavioral habituation, counterconditioning and persistence. In A. Black \& W. K. Prokasy (Eds.), Classical conditioning II. New York: Appleton-Century-Crofts, in press.

BACON, W. E. Resistance to extinction following blocking of the instrumental response during acquisition. Journal of Experimental Psychology, 1965, 69, 515-521.

BANKS, R. K. Intermittent punishment effect (IPE) sustained through changed stimulus conditions and through blocks of nonpunished trials. Journal of Experimental Psychology, $1967,73,456-460$.

BERLYNE, D. E. Conflict, arousal, and curiosity. New York: McGraw-Hill, 1960. Chaps. 6 and 7.

BROWN, J. S., \& BASS, B. The acquisition and extinction of an instrumental response under constant and variable stimulus conditions. Journal of Comparative \& Physiological Psychology, 1958, 51, 499-504.

BROWN, R.T., \& WAGNER, A. R. Resistance to punishment and extinction following training with shock or nonreinforcement. Journal of Experimental Psychology, 1964, 68, 503-507.

FALLON, D. Resistance to extinction following learning with punishment of reinforced and nonreinforced licking. Joumal of Experimental Psychology, 1968, 76, 550-557.

THOMPSON, R. F., \& SPENCER, W. A. Habituation: A model phenomenon for the study of neuronal substrates of behavior. Psychological Review, 1966, 73, 16-43.

NOTE

1. Supported by Grant No. GB-3772 from the National Science Foundation. We thank J. J. Hug for assistance in the statistical analysis. 